\title{
Caspase cleavage of cytochrome c1 disrupts mitochondrial function and enhances cytochrome c release
}

\author{
Yushan $\mathrm{Zhu}^{1,2,{ }^{*}}$, Min $\mathrm{Li}^{3,{ }^{*}}$, Xiaohui Wang ${ }^{1}$, Haijing $\mathrm{Jin}^{1}$, Shusen $\mathrm{Liu}^{1}$, Jianxin $\mathrm{Xu}^{3}$, Quan Chen ${ }^{1,2}$ \\ ${ }^{I}$ The Joint Laboratory of Apoptosis and Cancer Biology, The National Key Laboratory of Biomembrane and Membrane Biotech- \\ nology, Chinese Academy of Sciences, Beijing 100101, China; ${ }^{2}$ College of Life Sciences, Nankai University, Tianjin 300071, Chi- \\ na: ${ }^{3}$ Institute of Biophysics, Chinese Academy of Sciences, Beijing 100101, China
}

Mitochondrial catastrophe can be the cause or consequence of apoptosis and is associated with a number of pathophysiological conditions. The exact relationship between mitochondrial catastrophe and caspase activation is not completely understood. Here we addressed the underlying mechanism, explaining how activated caspase could feedback to attack mitochondria to amplify further cytochrome c (cyto.c) release. We discovered that cytochrome c1 (cyto.c1) in the bc1 complex of the mitochondrial respiration chain was a novel substrate of caspase 3 (casp.3). We found that cyto.c1 was cleaved at the site of D106, which is critical for binding with cyto.c, following apoptotic stresses or targeted expression of casp. 3 into the mitochondrial intermembrane space. We demonstrated that this cleavage was closely linked with further cyto.c release and mitochondrial catastrophe. These mitochondrial events could be effectively blocked by expressing non-cleavable cyto.c1 (D106A) or by caspase inhibitor z-VAD-fmk. Our results demonstrate that the cleavage of cyto.c1 represents a critical step for the feedback amplification of cyto.c release by caspases and subsequent mitochondrial catastrophe.

Keywords: cytochrome c1 cleavage; mitochondrial catastrophe; caspase; apoptosis

Cell Research (2012) 22:127-141. doi:10.1038/cr.2011.82; published online 17 May 2011

\section{Introduction}

Mitochondria are the intracellular powerhouse where energy substrates are metabolized to fuel the oxidative respiration reaction through the energy-transducing electron transport chain in the mitochondrial inner membrane. The oxidative respiratory chain consists of four multimeric complexes, which transfer electrons from the reducing substrates to oxygen to build up a proton gradi-

\footnotetext{
*These two authors contributed equally to this work.

Correspondence: Quan Chen ${ }^{\mathrm{a}}$, Jianxin $\mathrm{Xu}^{\mathrm{b}}$

${ }^{\mathrm{a}}$ Tel: +86-22-2350-2962

E-mail: chenquan@nankai.edu.cn

${ }^{\mathrm{b}} \mathrm{Tel}$ : +86-10-6487-1293

E-mail: xujx@sun5.ibp.ac.cn

Abbreviations: cyto.c (cytochrome c); cyto.c1 (cytochrome c1); SCR (succinate-cyto.c reductase); KD (knock-down); wt (wild type); STS (staurosporine); RCR (respiratory control ratio); NAC (N-Acetyl Cysteine); ATP (adenosine-triphosphate); Apaf-1 (apoptotic protease-activating factor 1); ROS (reactive oxygen species); casp.3 (caspase 3)

Received 20 January 2010; revised 17 March 2011; accepted 27 March 2011; published online 17 May 2011
}

ent for adenosine-triphosphate (ATP) generation [1-3]. The cytochrome bcl complex is the central component of the oxidative respiratory reaction chain that oxidizes ubiquinol and reduces cytochrome c (cyto.c). The interaction between cytochrome c1 (cyto.c1) and cyto.c ensures the fast complex formation, optimal orientation and distance for electron transfer and also fast dissociation after electron transfer, which is critical for maintaining the electron flow and preventing the potential electron leak $[4,5]$. The cytochrome bc 1 complex is also the major pathway for free radical generation within mitochondria [6-8]. Disruption of this interaction results in the loss of the oxidative phosphorylation, ample generation of reactive oxygen species (ROS) and disruption of mitochondrial functions and physiology leading to cell death. These mitochondrial metabolic catastrophes are causally associated with apoptosis at the cellular level and diseases in the pathophysiological conditions [9-11].

Mitochondrial catastrophe could be both the cause and consequence of apoptosis. Mitochondria sense the metabolic catastrophic signals and commit cells to apoptosis by releasing death-inducing factors such as cyto.c into 
the cytosol due to the permeabilization of mitochondrial outer membrane $[12,13]$. The release of cyto.c could result in mitochondrial respiration failure and the loss of mitochondrial functions. Once released into the cytosol, cyto.c binds to apoptotic protease-activating factor 1 (Apaf-1) to form a complex termed the apoptosome [1417], which in turn leads to the activation of downstream caspases and subsequent cleavage of cellular substrates at a specific tetra-peptide sequence on the carboxyl side of aspartate residues. Interestingly, it has been shown that mitochondrial catastrophes, including the loss of mitochondrial membrane potential following cyto.c release and the burst of ROS production, depend on the caspase activation in some systems [18-22]. Both pro- and activated caspases have been found in the mitochondrial intermembrane space [23-25], where they may disrupt mitochondrial function at the site of complex I and II [26, 27]. We previously showed that there were two distinct stages of cyto.c release into the cytosol during apoptosis and the activated caspases could cause further cyto. c release [4, 28, 29]. However, the molecular targets of caspase 3 (casp.3) and the precise mechanism of the amplification of cyto.c release by caspases are poorly understood. Here we report that cyto.c1 in the bc1 complex could be a novel target of casp. 3 both in vitro and in cells. This cleavage of cyto.c1 is critical for the amplification of cyto.c release and mitochondrial dysfunction. Our results are the first to show that the cleavage of cyto. $\mathrm{c} 1$, a key partner for cyto.c, results in further amplification of cyto.c release and subsequent mitochondrial catastrophe.

\section{Results}

Recombinant casp.3 inhibits mitochondrial respiratory activity

We previously observed that activated casp. 3 can induce cyto.c release, a rapid increase of ROS and the loss of integrity of isolated mitochondria [4]. We here specifically determined if recombinant human casp. 3 could disrupt the mitochondrial respiratory chain responsible for electron transfer and the mitochondrial ROS production. We first examined the effects of casp. 3 on the succinatecyto.c reductase (SCR) and cyto.c oxidase activity. Mitochondria isolated from mouse liver were incubated with or without casp. 3 at $37^{\circ} \mathrm{C}$ for various times, the activities of SCR and cyto.c oxidase were measured by the reduction or oxidation rate of exogenous cyto.c, respectively. Our result showed that recombinant casp. 3 significantly inhibited the SCR activity in a time-dependent manner (Figure 1A) and strongly induced the spontaneous generation of ROS in mitochondria in the presence of succinate (Figure 1B). The $\mathrm{H}_{2} \mathrm{O}_{2}$ generation from mitochondria was measured using the method of luminol as previously described. This event could be inhibited by zVAD-fmk, a pan caspase inhibitor (Figure 1B). Although recombinant casp. 3 has no effect on the activity of complex IV (Figure 1C), it potently inhibits the activity of complex III as measured by reduction of oxidized cyto. c (Figure 1D). Taken together, we conclude that complex III could be specifically targeted by recombinant casp.3.

\section{Identification of cyto.c1 as a casp. 3 substrate}

In order to identify the potential substrate of casp. 3 in the complex III, we purified SCR from pig hearts, in which the enzyme is highly conserved [30]. The complex III was treated with recombinant casp. 3 and Coomassie blue-stained diagonal gels were used to resolve the cleavage product, followed by SDS-PAGE analysis in the second dimension $[18,26]$. The two spots with a molecular size of about 10 and $25 \mathrm{kD}$ as indicated in Figure 2A were further analyzed by MALDI-TOF-MS. Both were identified as cyto.c1, a key partner of cyto. c for the electron transfer in the mitochondrial respiratory chain and a highly conserved protein from yeast to human. To confirm that cyto.c1 is indeed a substrate of casp. 3 , the mouse liver mitochondria were incubated with recombinant casp. 3 and the cleaved products were determined by western blotting analysis (Figure 2B). We next examined if endogenous cyto.c1 cleavage occurs in HeLa cells undergoing apoptosis. Cyto.c1 was found to be cleaved at $6 \mathrm{~h}$ after casp. 3 was activated at $3 \mathrm{~h}$ in the cells treated with staurosporine (STS) (Figure 2B). The casp.3-deficient mouse embryonic fibroblast (MEF) also demonstrated that casp. 3 is responsible for the cleavage of endogenous cyto.c1 and decreased apoptosis (Supplementary information, Figure S1).

As shown in Figure 2B, casp. 3 was able to cleave cyto.c1 at the $\mathrm{C}$-terminus to produce a peptide around $25 \mathrm{kD}$ and this cleavage could be completely inhibited by $\mathrm{z}$-VAD-fmk. Based on the sequence analysis (Figure 2C), several highly conserved Asp amino acid residues in cyto.c1 were mutated to Ala and the caspase cleavage analysis revealed that only the mutant with Asp mutation at residue 106, not at residues 128 and 157, was resistant to casp. 3 cleavage under the same experimental conditions (Figure 2D), suggesting that D106 is the specific cleavage site of casp.3. Although it is not a typical casp.3 cleavage site, it is interesting to note that this site is exposed to the intermembrane space and close to the heme and cyto.c-binding sites [34]. These data implicates that the cleavage of cyto.c1 by casp. 3 may occur in the intermembrane space and could have a profound impact on mitochondrial functions and apoptosis. 
A

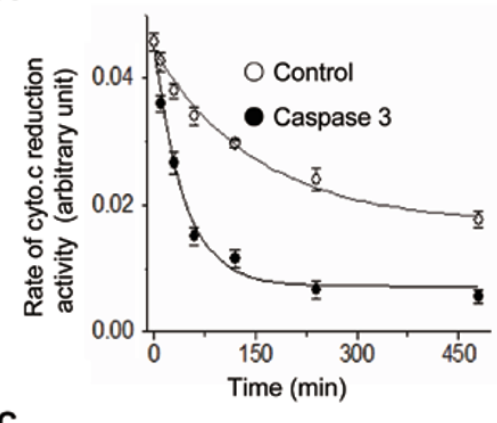

C

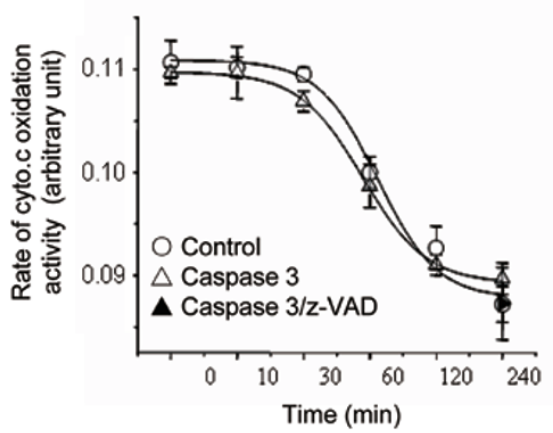

B

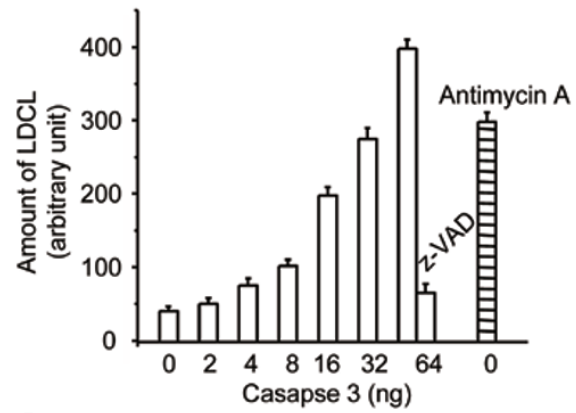

D

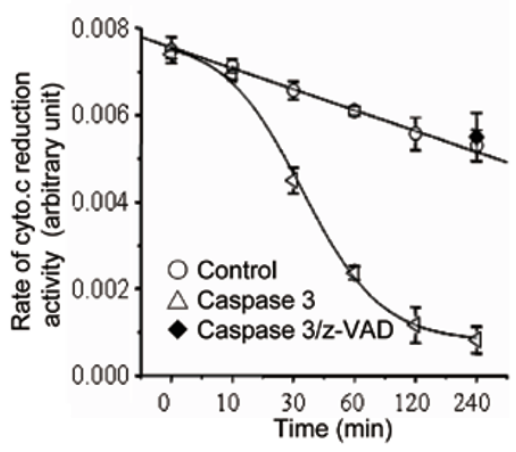

Figure 1 Recombinant casp.3 disrupts mitochondrial functions and physiology in vitro. (A) The activity of SCR was decreased by casp. 3 treatment in a time-dependent manner. SCR from mouse liver was incubated with or without recombinant casp.3 at $37{ }^{\circ} \mathrm{C}$ for the indicated times. The SCR activities were then measured by the reduction rate of cyto.c. (B) The effects of activated recombinant casp. 3 on mitochondrial ROS generation. Mitochondria extracted from mouse liver were incubated with different dosage of casp. 3 at $37^{\circ} \mathrm{C}$ for $1 \mathrm{~h}$ and the mitochondrial ROS generation was detected by LDCL. Antimycin A was used as a positive control. (C) The activated recombinant casp. 3 has no effect on the complex IV activity. Mitochondria extracted from mouse liver were incubated with or without casp. 3 at $37^{\circ} \mathrm{C}$ for the indicated times and the complex IV activity was detected by the oxidation of endogenous cyto.c. (D) The activated recombinant casp. 3 reduces the complex III activity. Mitochondria extracted from mouse liver were incubated with or without casp. 3 at $37^{\circ} \mathrm{C}$ for the indicated times and by using reduced quinine as the substrate, the complex III activity was demonstrated by the reduction of endogenous cyto.c. The data were the representative or mean value of at least 3 separate experiments.

Conversion of D106 to A in human cyto.c1 prevents STSinduced apoptosis and cyto.c release

In light of the importance of the interaction of cyto.c1 with cyto.c for the electron transfer and ROS generation, we determined the consequence of cyto.c1 cleavage in mitochondrial functions and apoptosis. Cells expressing wt (wild type) and non-cleavable form (D106 to A) human cyto.c1 were selected by Zeocin. As shown in Figure $3 \mathrm{~A}$ and $3 \mathrm{~B}$, the cells expressing the non-cleavable form of cyto.c1 were clearly resistant to STS-induced apoptosis. Both casp. 3 activation and cyto.c release were observed between 3-6 $\mathrm{h}$ after STS treatment as monitored by immunoblotting analysis (Figure 3B and 3C) and time lapse imaging of activated caspases (Supplementary information, Data S1, Figure S2 and Video S1A). Importantly, these events could be completely inhibited in the non-cleavable cyto.c1 or z-VAD-fmk. (Figure 3A-3C and Supplementary information, Figure S2, Video S1B and
S1C).

As cyto.c1 is an important partner of cyto.c for mitochondrial respiration, its cleavage could strikingly affect the release of cyto.c from mitochondria, leading to the amplification of apoptotic cascade. To address this possibility, the release of cyto.c was detected by both immunofluorescence and cell fractionation methods. Our results showed that cyto.c was released from mitochondria starting at $3 \mathrm{~h}$ in the cells expressing wt cyto.c1. In contrast, the cyto.c release was completely abrogated in the cells expressing the non-cleavable form of cyto. c1 (Figure 3B). To further substantiate this observation, we took an approach to transfect GFP-tagged cyto.c into HeLa cells and monitored the cyto.c release following the treatment of death stimuli. Clearly, we showed that the cyto.c release from mitochondria become visible at about $5 \mathrm{~h}$ after the exposure of STS in wt cyto.c1 cells (Supplementary information, Video S2A). However, the 
A

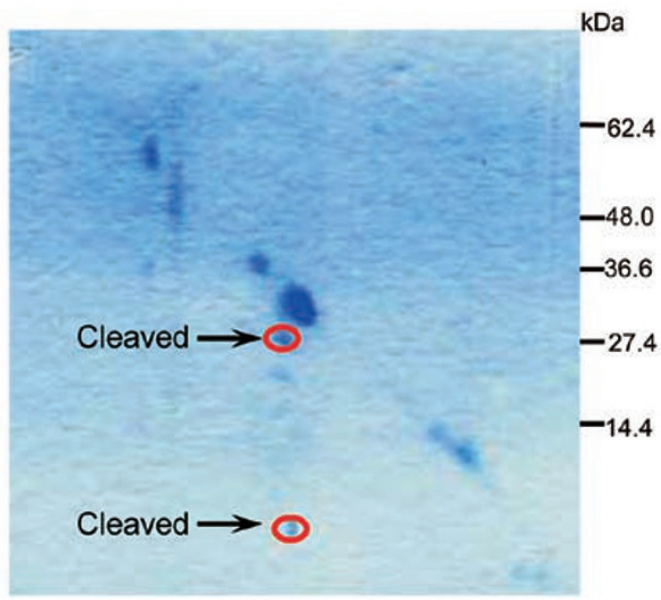

B

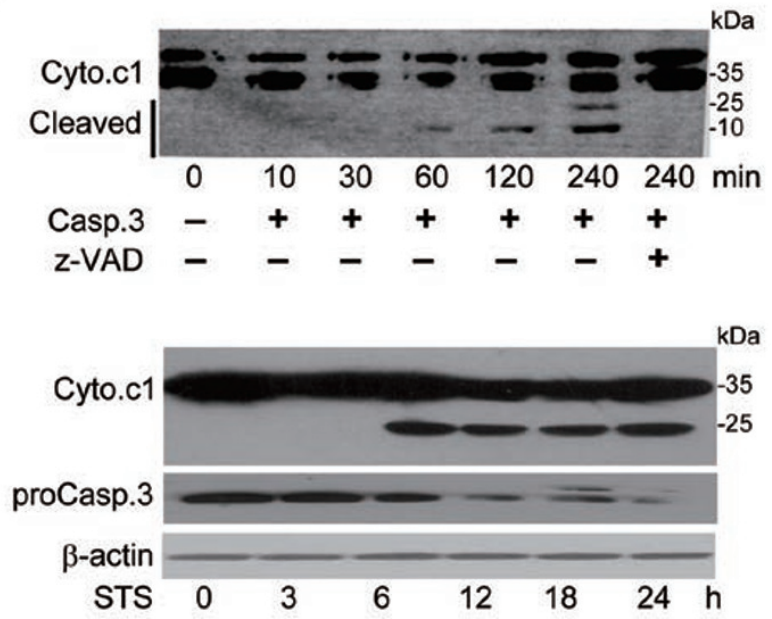

C
(81) 81

D106A

D128A

$120 \downarrow \downarrow 130$

140

D157A

Human (81) AVSASDLELHPPSYPWSHRGLISSIDHTS SRRGFQVYKQVCASCHSMDFVAYRHLVGVCYTEDEAKE LAAEVEVQDGPNED Bovine (81) AVSASDLELHPPSYPWSHRGLISSIDHTSIRRGEOVYKQVCSSCHSMDYVAYRHLVGVCYTEDEAKALAEEVEVQDGPNED

Mice (81) AVSASDLELHPPSYPWSHRGLLSSLDHTSIRRGFQVYKQVCSSCHSMDYVAYRHLVGVCYTEEEAKALAEEVEVQDGPNDD

Rat (22) AVSASDLELHPPSYPWSHRGLISSIDHTSIRRGFQVYKQVCSSCHSMDYVAYRHLVGVCYTEEEAKALAEEVEVQDGPNED

Yeast (61) AMTAAEHGLHAPAYAWSHINGPFETFDHASIRRGYQVYREVCAACHSLDRVAWRT LVGVSHTNEEVRNMAEEFEYDDEPDEC

Consensus (81) AVSASDLELHPPSYPWSHRGLLSSLDHTSIRRGFQVYKQVCSSCHSMDYVAYRHLVGVCYTEEEAKALAEEVEVQDGPNED

D
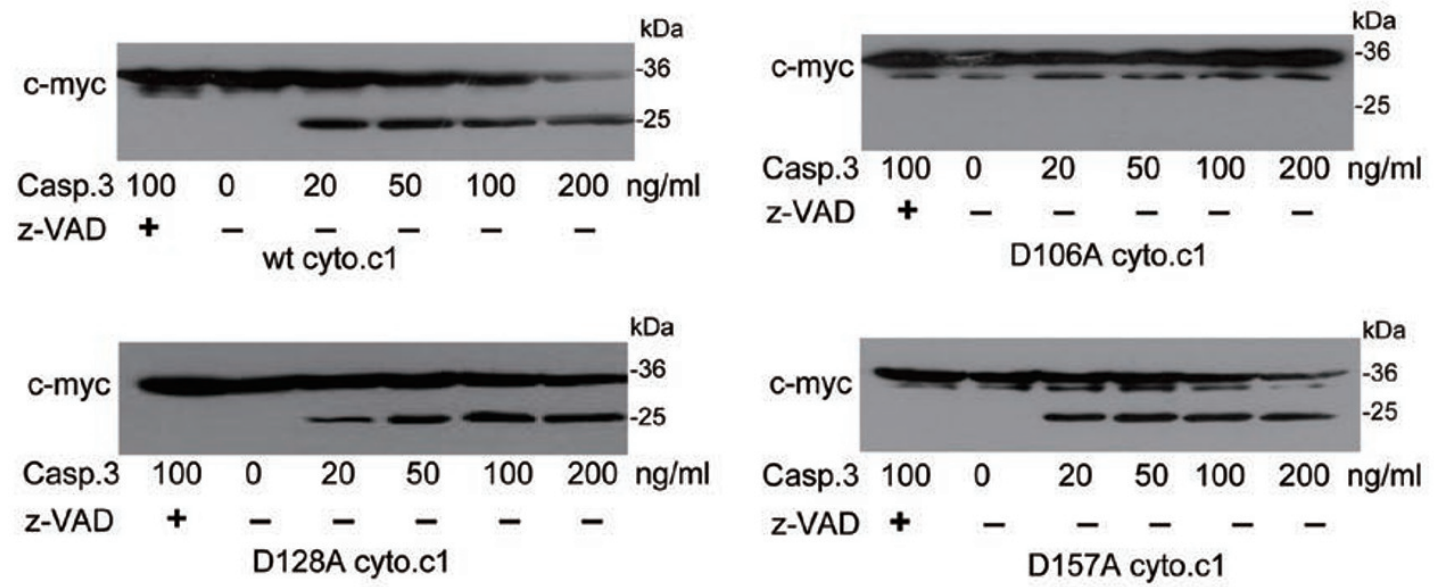

Figure 2 Identification of cyto.c1 as a novel casp.3 substrate. (A) Coomassie-stained diagonal gels analysis reveals that a component of SCR is cleaved by recombinant casp.3. SCR from swine heart were resolved by SDS-PAGE, and the lane was rehydrated in the presence of recombinant casp. 3 and resolved by SDS-PAGE in the second dimension. The two arrows indicate the spots corresponding to cyto.c1 as determined by MALDI-TOF-MS. (B) Cyto.c1 as a novel casp.3 substrate. Upper panel: mitochondria extracted from mouse liver were incubated with casp. 3 at $37^{\circ} \mathrm{C}$ for the indicated times in the presence or absence of z-VAD-fmk. Cyto.c1 cleavage was assessed by western blotting analysis. Lower panel: HeLa cells were treated with STS and the cell lystes were subjected to western blotting with anti-cyto.c1 or anti-casp.3 antibody. (C) Sequence alignment of the evolutionally conserved amino acids of cyto.c1 from several species. Residues that are identical and similar among the cyto.c1 are denoted in yellow and blue, respectively. The mutation sites are indicated by arrows. (D) Identification of D106 as a cleavage site. A volume of $20 \mu \mathrm{g}$ of isolated mitochondria from wt cyto.c1, D106A, D128A and D157A cells were treated with $200 \mathrm{ng}$ of activated recombinant casp. 3 at $37^{\circ} \mathrm{C}$ for $2 \mathrm{~h}$ in $50 \mu \mathrm{l}$ of reaction buffer in vitro. The reactions were stopped and resolved by $15 \%$ SDS-PAGE and subjected to western blotting with anti-myc antibody. 
A

B
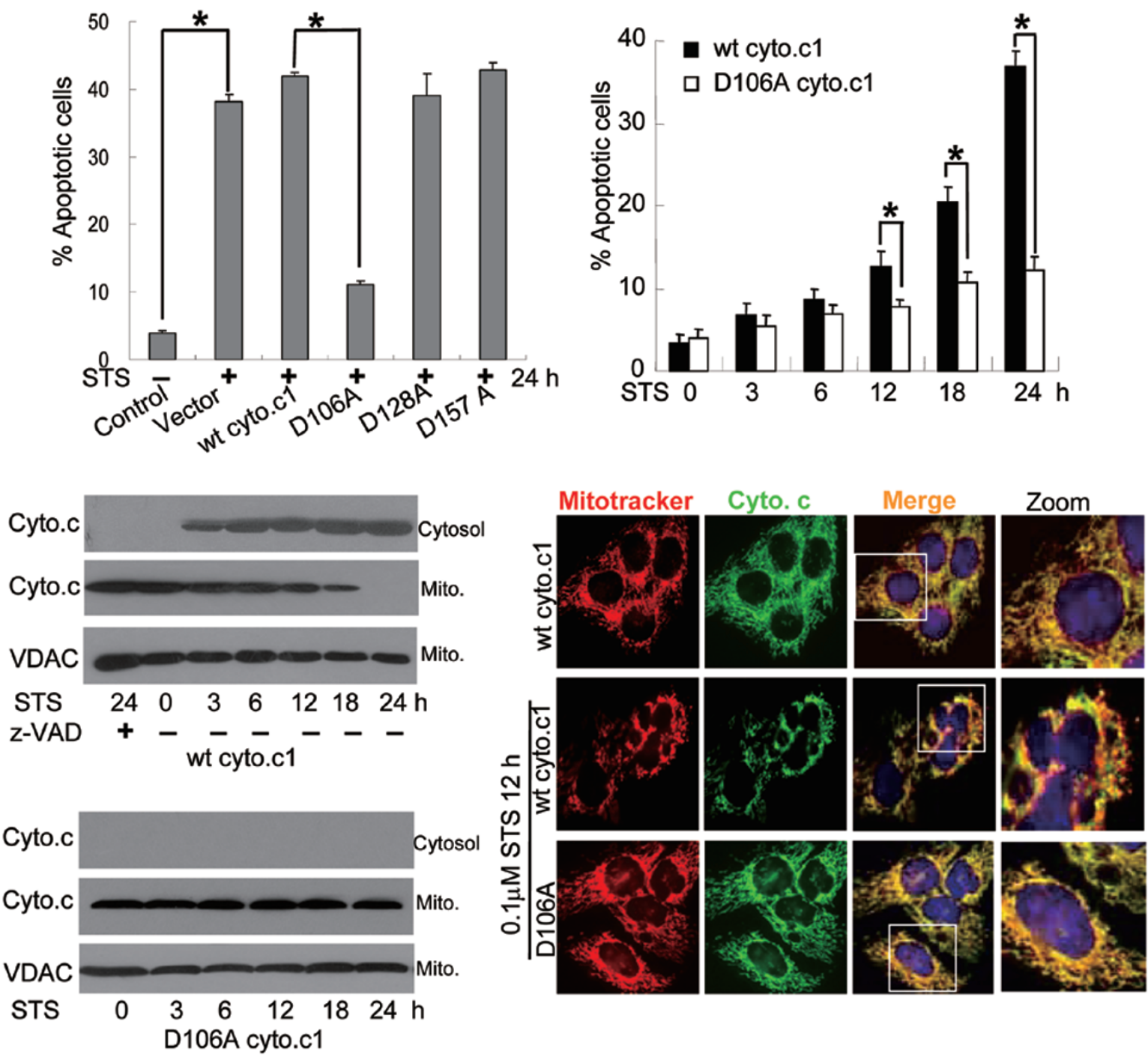

C
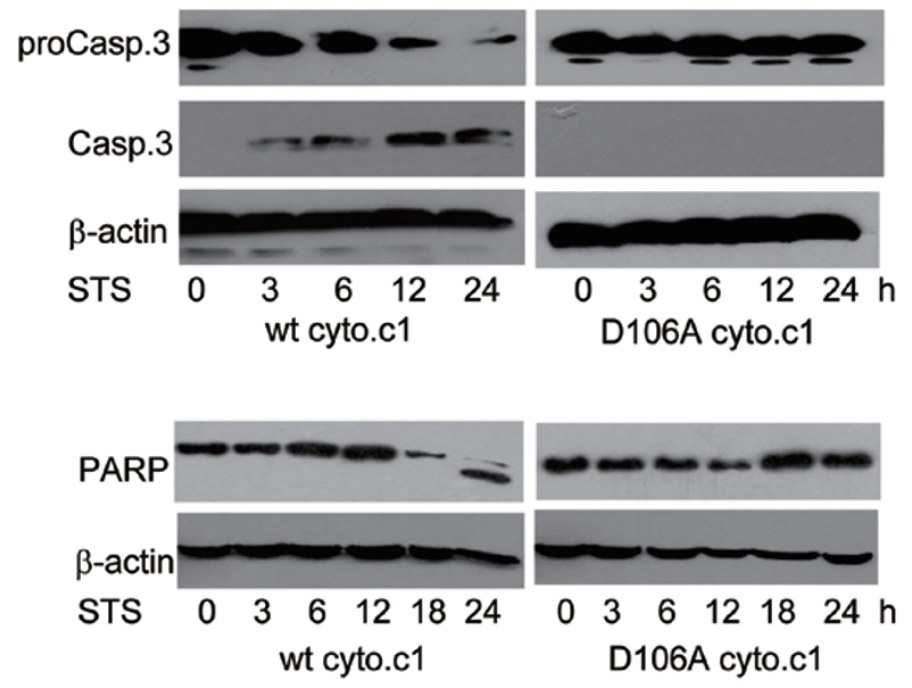

D
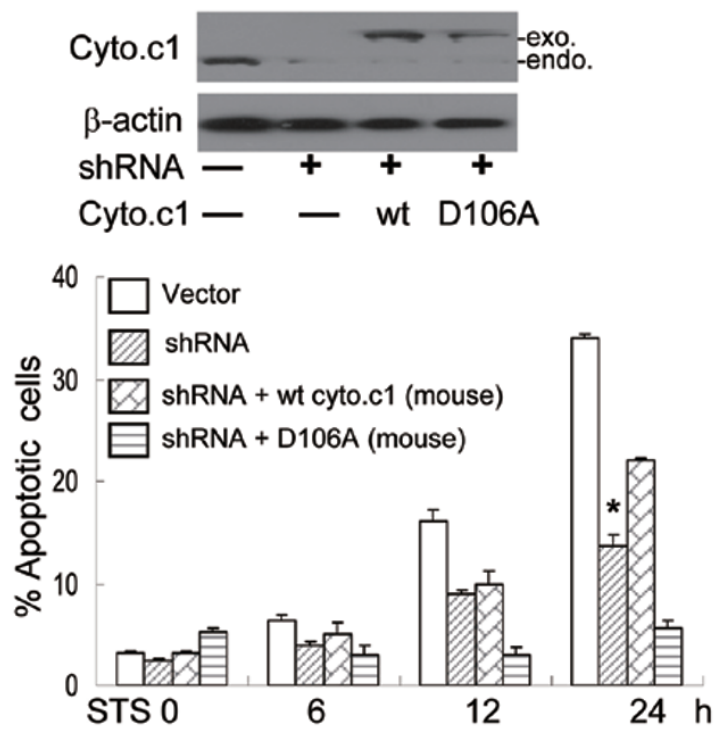
Figure 3 Non-cleavable cyto.c1 abrogates STS-induced apoptosis and cyto.c release. (A) Non-cleavable cyto.c1 prevents STS-induced apoptosis. Left: flow cytometric analysis of apoptosis in cells expressing different mutant cyto.c1 following treatment with STS for $24 \mathrm{~h}$. The percentage of Annexin V/PI positive cells was determined. Right: flow cytometric analysis of apoptosis in cells expressing wt and non-cleavable cyto.c1 exposed to STS $(0.1 \mu \mathrm{M})$ for the indicated times. (B) Noncleavable cyto.c1 prevents cyto.c release. Left: cells expressing wt or non-cleavable cyto.c1 were exposed to STS for the indicated times and subjected to subcellular fractionation. The cytosolic fraction (Cytosol) and mitochondrial fraction (Mito.) were analyzed by immunoblotting with anti-cyto.c antibody. Right: immunofluorescent microscopy of cyto.c release in wt and non-cleavable cyto.c1 cells treated with STS for 24 h. (C) Non-cleavable cyto.c1 blocks casp. 3 activation and PARP cleavage. Cells expressing wt or non-cleavable cyto.c1 were treated with STS for the indicated times, followed by immuoblotting analysis with anti-casp.3 (upper) or anti-PARP antibodies (lower). (D) The effect of exogenous wt and non-cleavable cyto.c1 on cell death in cyto.c1 shRNA cells. The wt or non-cleavable mouse cyto.c1 was reintroduced into the endogenous cyto.c1knockdown HeLa cells and the protein level of cyto.c1 was detected (upper). Lower panel showed flow cytometric analysis of apoptosis exposed to STS. The data were the representative or mean value of at least three separate experiments and statistical significance was determined by Student's $t$-test, ${ }^{*} P<0.05$.

cyto.c release and mitochondrial fragmentation were largely inhibited in the cells expressing non-cleavable cyto.c 1 or by z-VAD-fmk (Figure 3B and Supplementary information, Video S2B and S2C). At the same time, the PARP cleavage was observed in the wt cyto.c1, but not in the non-cleavable cyto.c1 cells by STS (Figure 3C). These data thus identified that the cleavage of cyto.c1 is a critical step for cells to undergo apoptosis. This effect is specific, as mutation of D128 or D157 to alanine does not confer the resistance of cyto.c1 to casp. 3 cleavage (Figure 2D) and these cells are still sensitive to STSinduced apoptosis (Figure 3A).

To further ascertain the effect of cyto.c1 cleavage on apoptosis, we used shRNA to specifically knock down the endogenous cyto.c1 and then reintroduced the RNAiresistant wt or non-cleavable mouse cyto.c1 into the cells. Knockdown of the endogenous cyto.c1 resulted in apoptosis inhibition (Figure 3D). Expression of the mouse wt cyto.c1, but not the non-cleavable cyto.c1, in the shRNA-knocking down HeLa cells restored the cell sensitivity towards apoptosis (Figure 3D). These results strongly support that cyto.c1 is a substrate of casp. 3 in vivo and the cleavage of cyto.c1 is critical for the progression of apoptotic phenotypes.

\section{HeLa cells expressing non-cleavable cyto.c1 maintain mitochondrial morphology and functions}

Mitochondria maintain their unique morphology for their functions and will undergo extensive morphological changes including mitochondrial swelling, remodeling of inner membrane and fragmentation during apoptosis. A line of evidence has suggested that these changes could be caspase dependent although the underlying mechanism is not clear. We thus examined if the cyto.c1 cleavage is associated with mitochondrial morphology and functions. As shown in Figure 3B (right panel), STS was able to effectively induce the fragmentation of mitochondria in HeLa cells expressing wt, not non-cleavable cyto.c1. In addition, we found that the mitochondrial fragmentation could be inhibited by $N$-acetyl cysteine (NAC) (Supplementary information, Figure S3) suggesting that ROS, likely to be generated from the impaired mitochondrial respiratory chain, involves mitochondrial fragmentation.

We next examined the effects of the expression of wt and non-cleavable cyto.c1 on mitochondrial respiration during apoptosis. Upon STS treatment, the state IV respiration was maintained while there was significant reduction of state III respiration. The respiratory control ratio (RCR) in the cells expressing wt, but not non-cleavable cyto.c1, was remarkably decreased in the presence of STS. Interestingly, the ATP synthase activity in both cell types was decreased, but the cellular NADH levels in both cells were not affected upon STS treatment. Therefore, it seemed that the coupling of mitochondrial electron transfer and ATP generation was compromised while the cleavage of cyto.c1 was occurring. Accordingly, noncleavable cyto.c 1 prevented the loss of mitochondrial physiology and functions to maintain their viability when treated with STS (Table 1). These results demonstrated that the cleavage of cyto.c1 resulted in the metabolic catastrophe.

To further elucidate the effect of the interaction between cyto.c1 and cyto.c on the cyto.c release, we used rho zero cells, which are deficient in mtDNA and lack electron transfer and ROS production in mitochondria. Interestingly, we were still able to detect casp. 3 activation and its cleavage of cyto.c1 (Supplementary information, Figure S4), suggesting that the cyto.c release could occur in the absence of mitochondrial respiration and ROS. This further supports that the cleavage of cyto.c1 is important for cyto.c release. Collectively, these results demonstrated that the cleavage of cyto.c1 at D106 is critical for cyto.c release, which may represent the critical step for the release of cyto.c positively feedback to attack mitochondria to amplify cyto.c release as we previously 
Table 1 Effect of the mitochondrial respiratory, cell oxidative phosphorylation and aerobic glycolysis by STS

\begin{tabular}{|c|c|c|c|c|c|c|}
\hline & $\begin{array}{l}\text { RCR } \\
\text { (control) }\end{array}$ & $\begin{array}{l}\mathrm{RCR} \\
(\mathrm{STS})\end{array}$ & $\begin{array}{l}\text { ATP synthase activity } \\
\text { (control) }\end{array}$ & $\begin{array}{l}\text { ATP synthase activity }{ }^{1} \\
(\mathrm{STS})\end{array}$ & $\begin{array}{l}\mathrm{NADH}^{2} \\
\text { (control) }\end{array}$ & $\begin{array}{l}\mathrm{NADH}^{2} \\
(\mathrm{STS})\end{array}$ \\
\hline Mock & $2.8 \pm 0.3$ & $1.6 \pm 0.5$ & $108 \pm 8.1$ & $63 \pm 6.7$ & $1.2 \pm 0.08$ & $1.0 \pm 0.20$ \\
\hline D106A Cyt.C1 & $3.5 \pm 0.2$ & $3.2 \pm 0.3$ & $123 \pm 10.1$ & $82 \pm 4.9$ & $1.6 \pm 0.14$ & $1.6 \pm 0.21$ \\
\hline
\end{tabular}

Cells were cultured in the presence or absence of STS $(0.1 \mu \mathrm{M})$ for $12 \mathrm{~h}$ and collected. The cellular NADH level and mitochondrial ATP synthase activity were detected as described in Materials and Methods. Data are expressed as mean \pm SEM.

${ }^{1} \mathrm{nmol} / \mathrm{min}$ per mg protein

${ }^{2}$ Fluorescence (arbitory units) $/ 10^{6}$ cell.

A

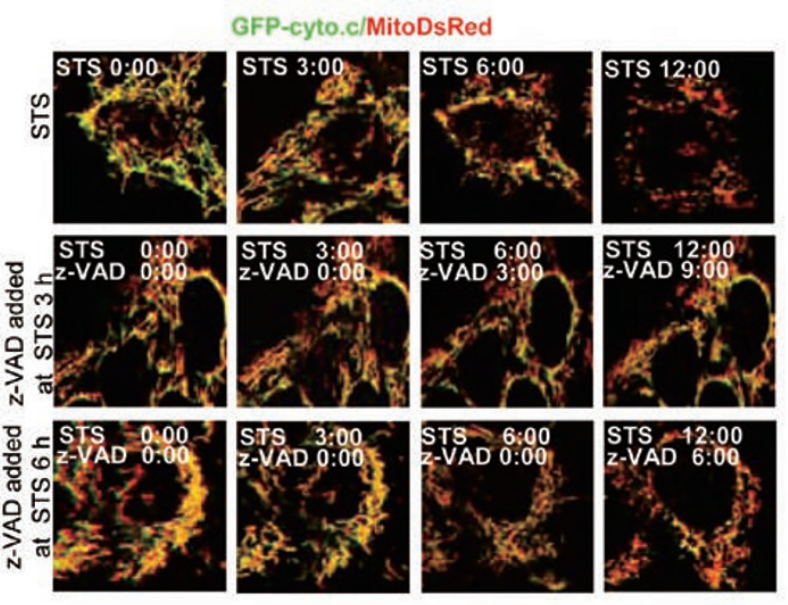

B

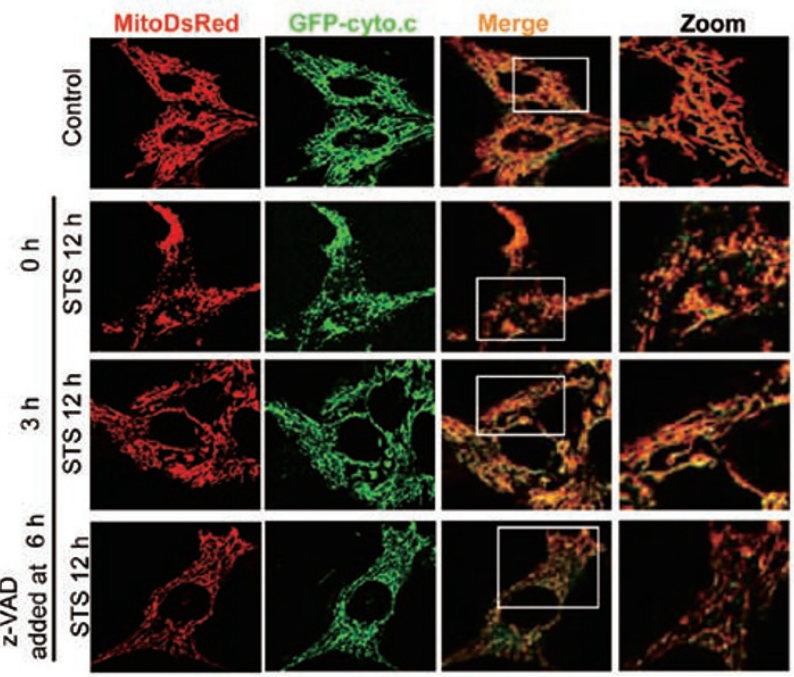

D
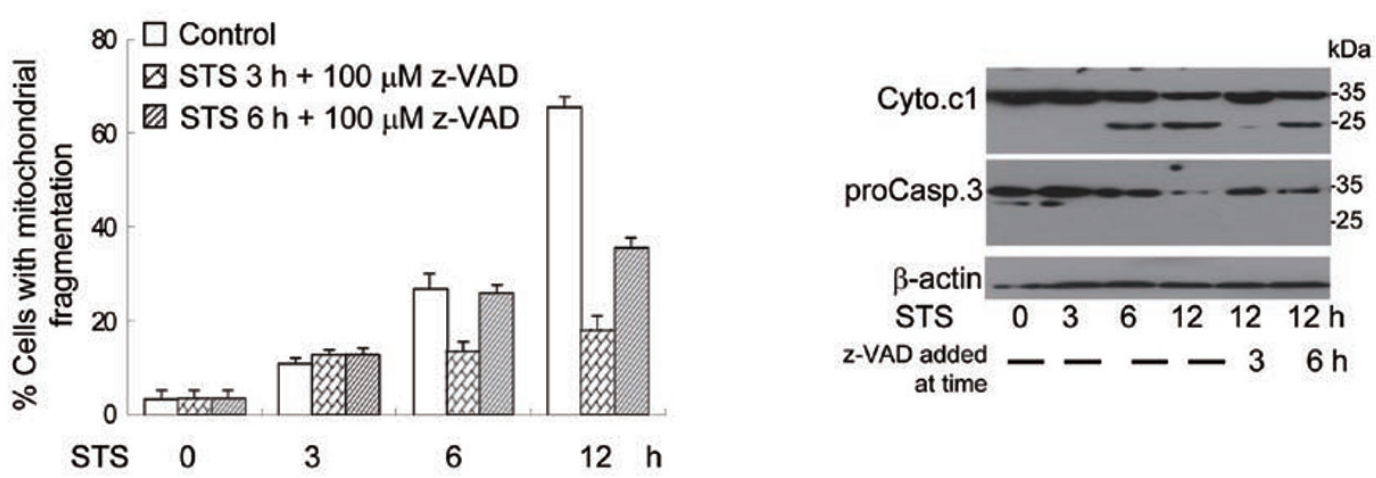

Figure 4 The z-VAD-fmk prevents cyto.c1 cleavage and inhibits cyto.c release and mitochondrial fragmentation. (A) Representative images from the video recording showing that z-VAD-fmk inhibits cyto.c release when added at 3 or $6 \mathrm{~h}$ after STS treatment. The GFP-cyto.c/MitoDsRed cells were treated with STS for 0,3 and $6 \mathrm{~h}$ and z-VAD-fmk (100 $\mu \mathrm{M})$ was added at the indicated times. Time lapse video of cyto.c levels and mitochondrial morphology were conducted for $12 \mathrm{~h}$. Cells treated with STS alone for $12 \mathrm{~h}$ (upper). Cells supplemented with z-VAD-fmk after treated with STS for $3 \mathrm{~h}$ (middle). Cells supplemented with z-VAD-fmk after treated with STS for $6 \mathrm{~h}$ (lower). (B, C) GFP-cyto.c/MitoDsRed cells were first treated by STS for 0 , 3 and $6 \mathrm{~h}$ and then supplemented with z-VAD. Cells were fixed and analyzed by confocal microscopy at $12 \mathrm{~h}$ to determine cyto.c levels and mitochondrial morphology. (D) The z-VAD-fmk partially inhibits cyto.c1 cleavage and casp. 3 activation when added at later time points. HeLa cells were treated with $0.1 \mu \mathrm{M}$ STS for the indicated times and then supplemented with zVAD-fmk before harvesting at $12 \mathrm{~h}$. Both cyto.c1 cleavage and casp. 3 were detected by western blotting. 
proposed.

z-VAD-fmk inhibits cyto.c release and mitochondrial fragmentation even when casp. 3 is partially activated

Our data collectively have shown that cyto.c1 cleavage by casp. 3 is important for the apoptotic events such as cyto.c release and mitochondrial fragmentation. To test that there is a positive feedback loop for caspase amplification of cyto.c release via attacking mitochondria, we treated the cells with STS for 3 or $6 \mathrm{~h}$, which allows certain levels of activation of caspases and then monitored the cyto.c release in the presence or absence of z-VAD-fmk (Figure 4). Although casp. 3 was activated at 3 and $6 \mathrm{~h}$ in the cells, z-VAD-fmk can still largely block further cyto.c release (Figure 4 and Supplementary information, Videos S2 and S3). Mitochondrial fragmentation was also inhibited by z-VAD-fmk, even 3 and $6 \mathrm{~h}$ after STS treatment (Figure 4 and Supplementary information, Video S3B and S3C). These data strongly suggest that caspase could feedback attack mitochondria for the progression of apoptosis.

Truncated cyto.cl enhances mitochondrial fragmentation

To address if the truncated product of cyto.c1 affects mitochondrial functions and cyto.c release, we transiently expressed myc-tagged wt cyto.c1, truncated cyto.c $1^{1-}$ ${ }^{106}$ and cyto.c $1^{107-325}$ in HeLa cells. As expected, wt cyto. c1 does not affect mitochondrial morphology. Strikingly, the truncated cyto.c $1^{1-106}$ alone enhanced mitochondrial fragmentation (Figure 5A and 5B) as shown by significantly less staining of mitotracker, indicative of the loss of mitochondrial membrane potential [31]. These cells are also more sensitive to STS-induced mitochondrial fragmentation and membrane potential loss (Figure 5A and $5 \mathrm{~B}$ ). Furthermore, we also found that the truncated cyto.c $1^{1-106}$-induced cells death and cyto.c release in the cyto.c1 knock-down (KD) cells (Figure 5C and 5D). However, the truncated cyto.c $1^{107-325}$ failed to localize at mitochondria (as it lacks mitochondrial-targeting sequence) and had no effect on mitochondrial morphology (data not shown). Therefore, our data clearly showed that truncation of cyto.c1, which affects the interaction of endogenous cyto.c1 with cyto.c, resulted in the impaired mitochondrial respiration and increased ROS for mitochondrial fragmentation.

Targeted expression of casp. 3 into mitochondrial intermembrane space resulted in cyto.cl cleavage and cyto.c release

To demonstrate that activated casp. 3 in the mitochondrial intermembrane space mediates the cyto.c1 cleavage and mitochondrial dysfunction, we carried out the targeted expression of wt casp. 3 into the intermembrane space of mitochondria with the aid of a signaling peptide from Smac (an intermembrane space protein) [32, 33]. Our results showed that targeted expression of the signaling peptide tagged-casp. 3 resulted in mitochondrial cyto.c release, leading to mitochondrial fragmentation, while expressing the signaling peptide alone had no effect on the mitochondrial morphology (Figure 6A-6C). Concomitantly, cyto.c1 was cleaved at $12 \mathrm{~h}$ after transfection when the activity of casp. 3 could be detected (Figure 6D). These events can be blocked by z-VADfmk, suggesting that the mitochondrial phenotypes were dependent on the activated casp. 3 in the intermembrane space. More importantly, mitochondria and cell death in cells with the non-cleavable cyto.c1 were not affected by the expression of mitochondria-targeted casp.3 (Figure $6 \mathrm{~B})$. These data strongly support that activated casp. 3 in the intermembrane space of mitochondria is responsible for the cyto.c1 cleavage and subsequent mitochondrial dysfunction and cell death.

\section{Discussion}

Our current study identified cyto.c1 as a novel substrate for casp.3. We demonstrated that the cleavage of cyto.c1 caused mitochondrial catastrophe as manifested by impaired mitochondrial ATP generation, the increase of ROS and profound mitochondrial fragmentation. The cleavage could disrupt the interaction of bc 1 complex with cyto.c and the electron transfer between bc1 complex and complex IV, resulting in further amplification of mitochondrial cyto.c release and subsequent metabolic catastrophe (Figure 6E). Our data clearly showed that mitochondrial dysfunction induced by caspase feedback attack is important for the progression of apoptosis.

Our results provide a new mechanism for how caspases feedback to amplify cyto.c release and the progression of apoptosis. Casp. 3 was activated and indeed translocated into mitochondria following apoptotic stresses. Intriguingly, targeted expression of casp. 3 in the mitochondrial intermembrane space could faithfully reproduce the mitochondrial catastrophic phenotypes and cause cyto.c1 cleavage and cyto.c release from mitochondria. Importantly, caspase inhibitor abrogated cyto.c1 cleavage and cyto.c release even when casp. 3 was partially activated. These results highlight the importance for the feedback mechanism for cyto.c release and subsequent mitochondrial dysfunctions. It was reported that cyto.c1 works as a homodimer in the bc1 complex [34]. Thus, cleavage of both molecules of cyto.c1 in the same complex would result in the loss of its physical interaction with cyto.c and mitochondrial functions. In addition, the cleavage of 
A
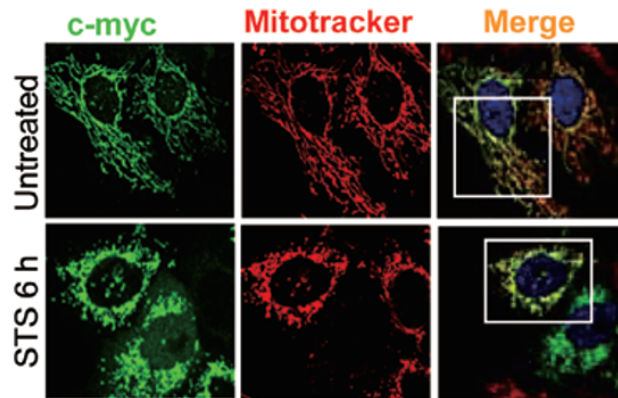

Zoom
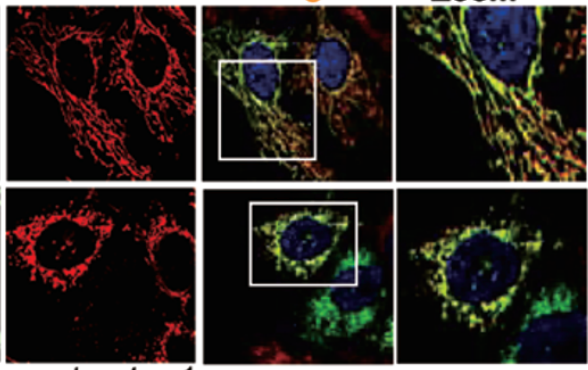

wt cyto.c
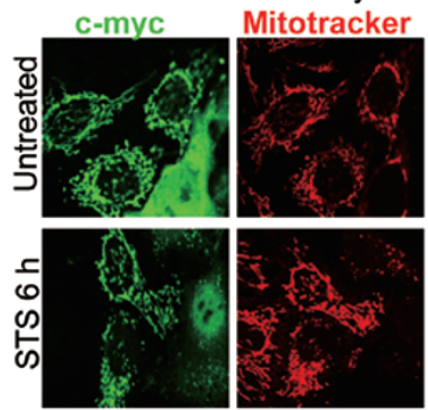

Truncated cyto.c1 (1-106)

B

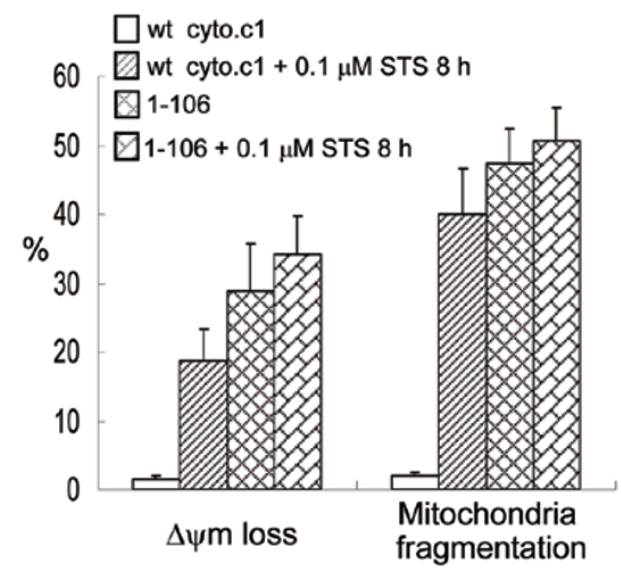

C
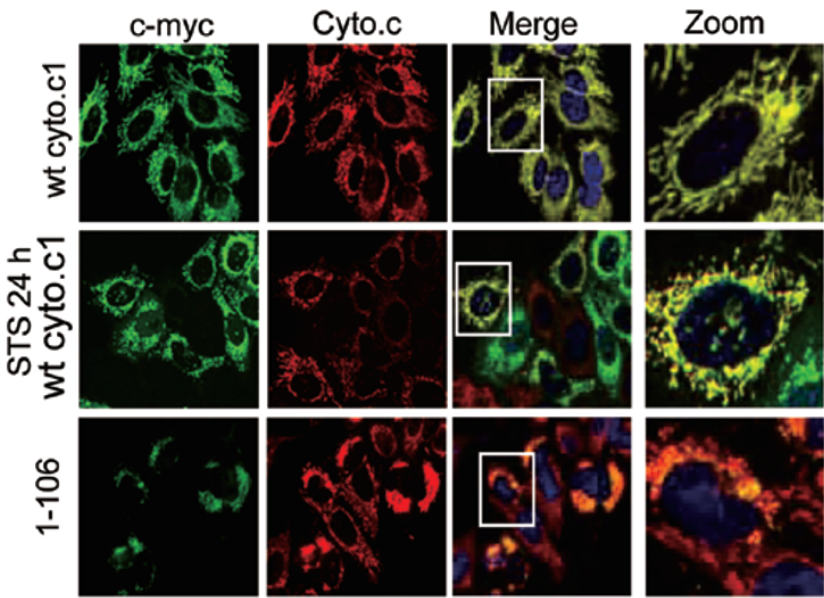

D
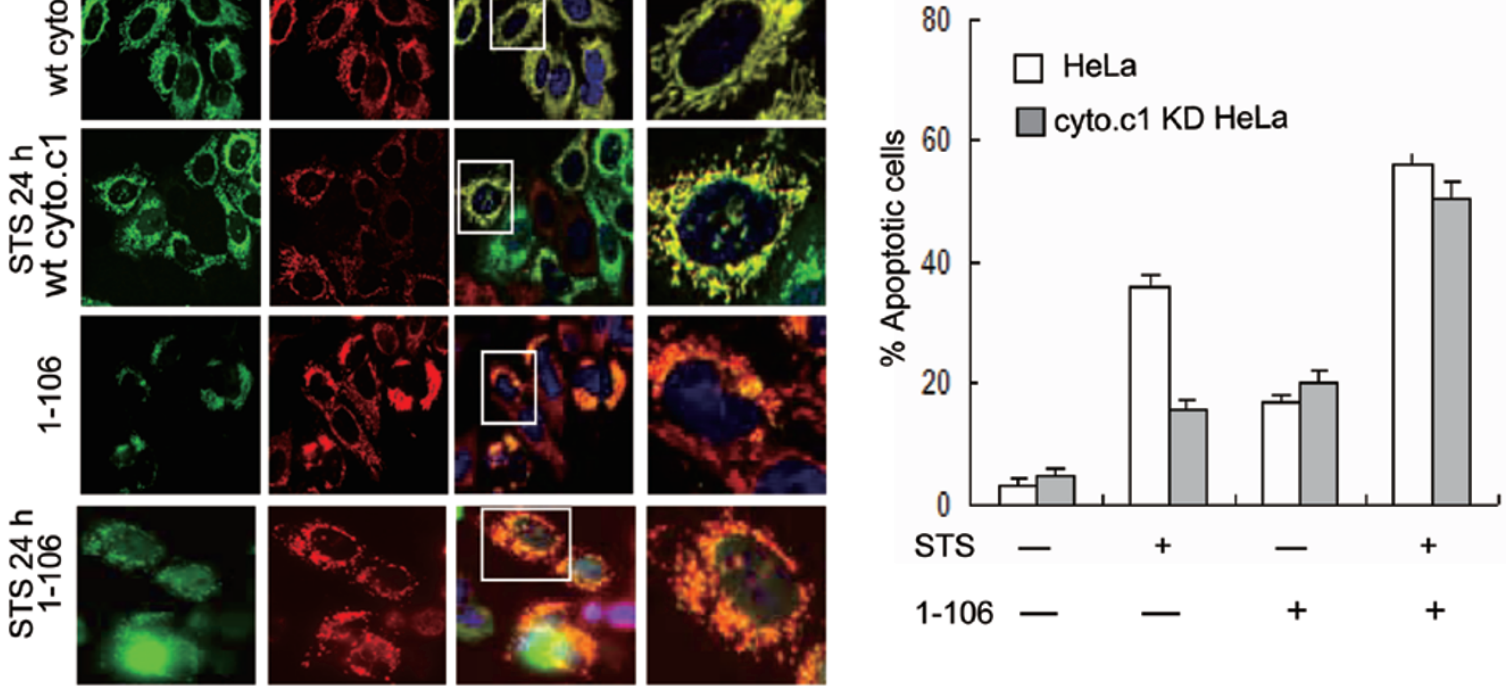

Cyto.c1 KD cells

Figure 5 Truncated cyto.c1 affects the mitochondrial morphology. (A) Cells were transfected with wt and truncated cyto.c1 and then exposed to $0.1 \mu \mathrm{M}$ STS for $6 \mathrm{~h}$. Cyto.c1 expression was detected using an anti-myc primary antibody and FITCconjugated secondary antibody. Mitochondria were visualized using mitotracker. Mitochondria in cells expressing truncated cyto.c1 are less stained with mitotracker (indicative for the loss of mitochondrial membrane potential) and become fragmented when exposed to STS for $8 \mathrm{~h}$. (B) Statistical results of membrane potential loss (as indicated by the inability to mitotracker staining) and mitochondrial fragmentation in wt and truncated cyto.c1 $1^{1-106}$ cells. (C) Cyto.c1 KD Cells were transfected with the mouse wt cyto.c1 or truncated cyto.c $1^{1-106}$ for $24 \mathrm{~h}$ and treated with STS for $24 \mathrm{~h}$; cyto.c1 expression and cyto.c release were detected using anti-myc (rabbit) and cyto.c (mouse) antibody, followed by incubating with goat anti-rabbit-FITC and donkey anti-mouse-CY3 secondary antibody, respectively. (D) Flow cytometric analysis of apoptosis (Annexin V/PI positive) in the cyto.c1 KD cells expressing wt and truncated cyto.c1 ${ }^{1-106}$ following treatment with STS $(0.1 \mu \mathrm{M})$ for $24 \mathrm{~h}$. 
A
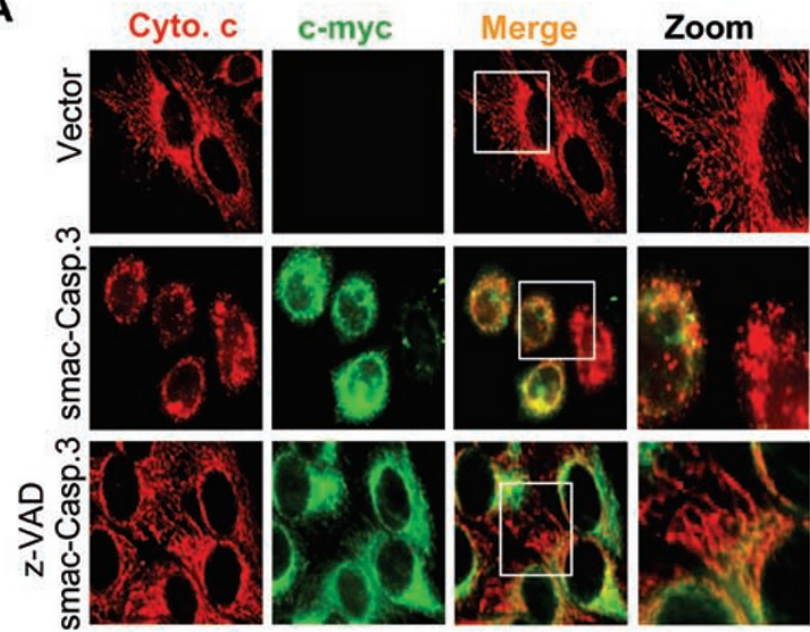

C
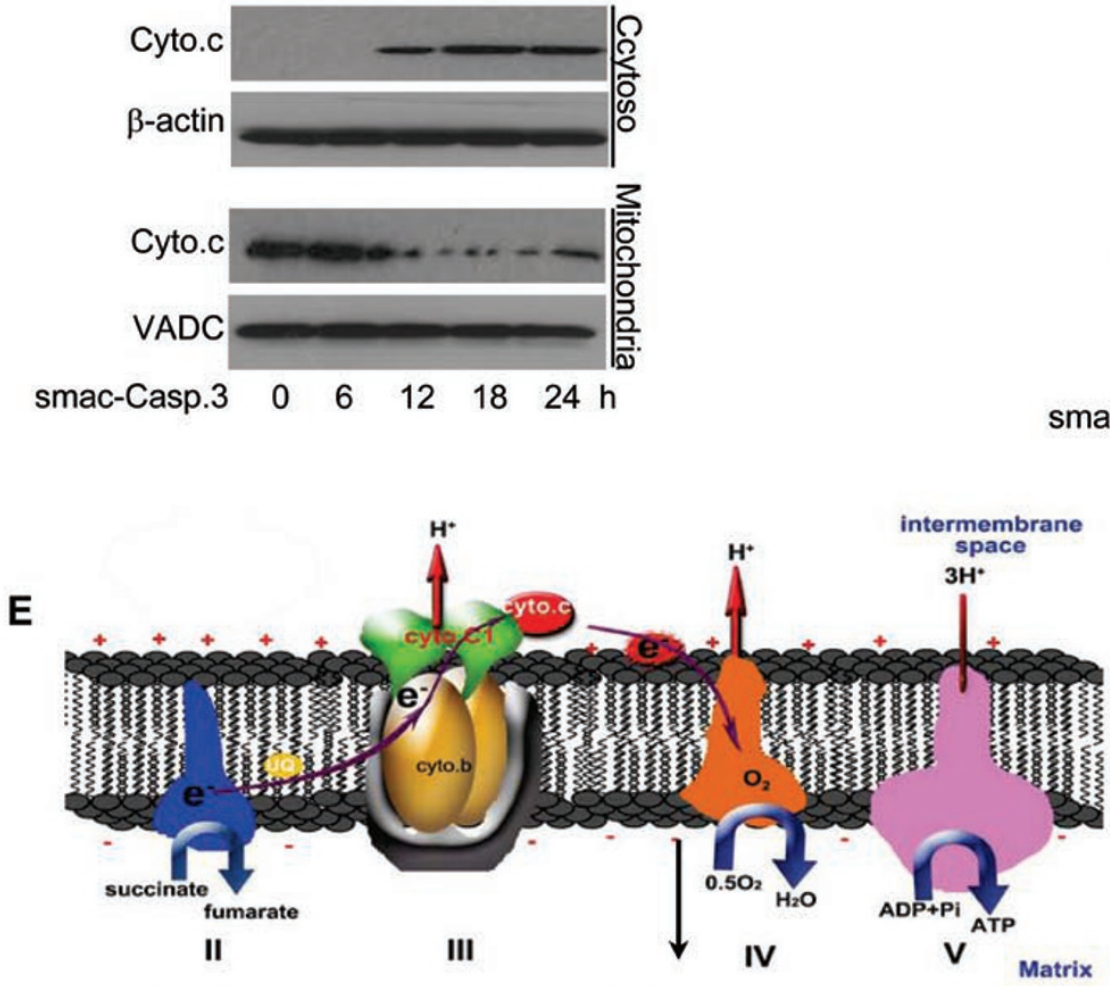

Membrane potential loss caspase 3 $0 ?$

IV

D

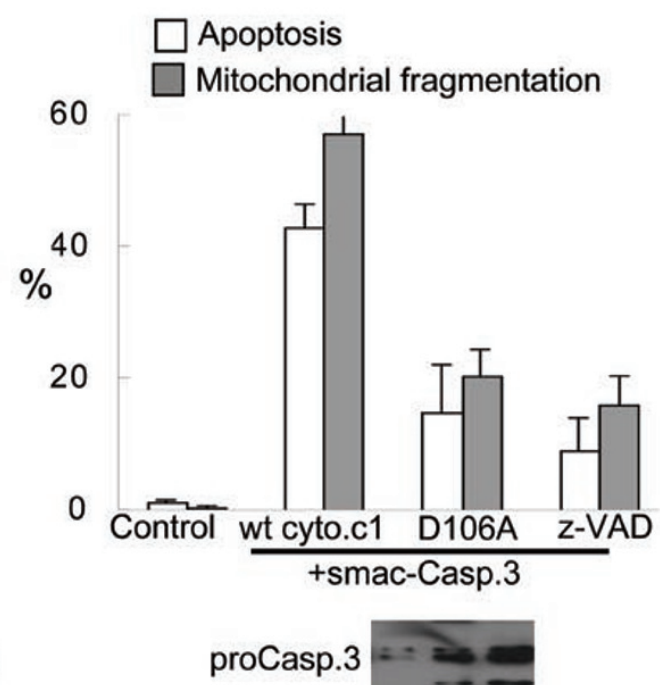

Casp. 3

Cyto.c1

Cleaved

$\beta$-actin

smac-Casp.3 $0 \quad 1212 \mathrm{~h}$

z-VAD - - +

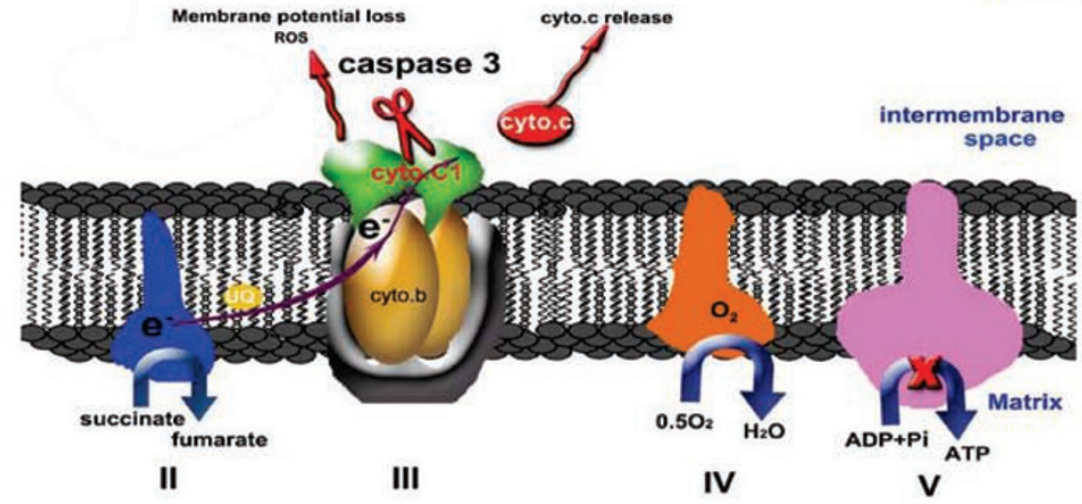


Figure 6 Targeted expression of casp. 3 into mitochondrial intermembrane space resulted in cyto.c1 cleavage and cyto.c release. (A) Cells were transfected with the Smac signaling peptide-tagged casp.3 (Smac-casp.3) in the absence or presence of z-AVD-fmk, fixed and Smac-casp.3 expression was detected by rabbit c-myc and a FITC-conjugated rabbit secondary antibody. (B) The z-VAD-fmk and non-cleavable cyto.c1 inhibited cell death and mitochondrial fragmentation. Statistical analysis showing that Smac-casp.3 induced cell death and mitochondrial fragmentation in the wt, non-cleavable cyto.c1 and HeLa cells pre-treated with z-AVD-fmk. (C) Smac-casp. 3 induced cyto.c release from mitochondria. Mitochondria and cytosol were isolated from cells transfected with Smac-casp. 3 for $0,6,12,18$ and $24 \mathrm{~h}$, and cell lyastes were subjected to immunoblotting analysis. (D) Smac-casp.3 induced cyto.c1 cleavage. Cells were transfected with Smac-casp. 3 in the absence or presence of 1 z-AVD-fmk for 12 h, casp. 3 activation and cyto.c1 cleavage were detected by anti-casp. 3 and anti-cyto.c1 antibody, respectively. (E) Model on how cyto.c1 cleavage by casp.3 affects cyto.c release. The cytochrome bc1 complex is the central component of the oxidative respiratory reaction chain that oxidizes ubiquinol and reduces cyto.c. Casp.3 cleavage of cyto.c1 disrupts electron transport chain leading to the increase of ROS, ample release of cyto.c, the loss of mitochondrial membrane potential and mitochondrial function for ATP generation (lower).

cyto.c1 could affect the respiration status, ROS production and redox potential of mitochondria that may also contribute to cyto.c release in normal cells $[35,36]$. It should be noted that reduced form of cyto.c functions as an electron acceptor and physically interacts with cyto. c1. Thus, redox status of cyto.c is important for the retention of cyto.c in place [37,38]. It is interesting to note that, upon apoptotic stimulation, apoptosis was induced and cyto.c1 was cleaved in rho zero cells, which are deficient in mitochondrial DNA-encoding cytochrome $b$ and other key components of bc 1 complex in the respiration chain and do not generate mitochondrial ATP or mitochondrial ROS $[39,40]$. Thus, the cyto.c1 cleavage is important for cyto.c release and cell death.

Previous reports have shown that caspases are located in the mitochondrial intermembrane space [21, 2325] and affect mitochondrial ROS production [41]. Our data for the first time suggest that the bc1 complex, the central component of the oxidative respiratory reaction chain and a center for free radical generation, could be targeted by casp.3. Several mitochondrial membrane proteins such as Bcl-2 and Mcl-1 have been identified to be cleaved by caspases, leading to the conversion of antiapoptosis molecules into a Bax-like death promoter and the permeabilization of outer membrane of mitochondria $[42,43]$. As a result, activated caspases are able to penetrate into the intermembrane space to cleave cyto.c 1 for subsequent mitochondrial catastrophe. Previous study has shown that other proteins in mitochondrial respiration complex are also subject to caspase attack. For example, a mitochondrial inner membrane protein termed as p75, a subunit of complex I, is cleaved by activated caspases when mitochondria become permeabilized [41]. The cleavage of $\mathrm{p} 75$ leads to increase of ROS and loss of plasma membrane integrity without affecting mitochondrial cyto.c release. These results demonstrate that the mitochondrial respiratory chain is one of the major targets by caspases for promoting apoptosis.
The mitochondrial bc1 complex is the major site for intracellular ROS production $[6,34]$ and genetic defects leading to mutations in the subunits of the bc 1 complex result in mitochondrial myopathies and other diseases [44, 45]. Mutation of bc1 could also affect the stability of mitochondrial respiration complex I [46]. Our results suggest that cyto.c1 is important for mitochondrial morphology and functions. Expression of truncated cyto.c $1^{1-106}$ could also affect mitochondrial phenotype. Knockdown of cyto.c1 by specific shRNA could reduce cell death in mammalian cells. Disruption of the interaction between cyto.c1 and cyto.c by caspase could impair mitochondrial functions and cause mitochondrial catastrophe. Both mitochondrial metabolic catastrophe and abnormal apoptosis are closely associated with a number of pathophysiological conditions such as diabetes, neurodegenerative diseases and cancers $[38,41]$. Our results offer a fresh understanding of how metabolic catastrophe is causally linked with apoptosis and better understanding of the interplay of these complex processes will be useful to fight these metabolic and aging-related diseases.

\section{Materials and Methods}

\section{Materials}

Antibodies: Anti-cyto.c and anti-PARP (BD56433, 56362), anti-cyto.c1(Protein Tech), anti- $\beta$-actin (Sigma, a5441), anti-myc (Santa Cruz, sc-40) and anti-casp.3 (Q Chen Lab).

Reagents: Caspase inhibitor z-VAD-fmk (CalBiochem 627610), CaspACETM FITC-VAD-fmk (Promega G7462), PhiPhiLuxG1D2 (Oncoimmunin A304R1G) and Annexin V-FITC kit (Q Chen Lab). All other chemicals were purchased from Sigma unless otherwise specified.

\section{Cell culture}

HeLa cells were maintained in DMEM medium supplemented with $10 \%$ FBS (Hyclone) and $1 \%$ penicillin-streptomycin at $37{ }^{\circ} \mathrm{C}$ and $5 \% \mathrm{CO}_{2}$. To establish cell lines stably expressing cyto.c1, HeLa cells were transfected with pCDNA4.0-cyto.c1/myc.his plasmid DNA by phosphate calcium and positive clones overexpressing 
cyto.c1 were selected with $100 \mu \mathrm{g} / \mathrm{ml}$ Zoecin (Invitrogen R25001).

\section{Isolation of mitochondria}

Mitochondria isolation from HeLa cells Cells $\left(5 \times 10^{6}\right)$ were washed twice with ice-cold PBS and kept on ice for $1 \mathrm{~h}$ in hypotonic buffer containing $20 \mathrm{mM}$ HEPES-KOH (pH 7.2), 100 mM KCl, $1.5 \mathrm{mM} \mathrm{MgCl}_{2}, 1 \mathrm{mM}$ EDTA, $1 \mathrm{mM}$ EGTA, $250 \mathrm{mM}$ sucrose and protease inhibitors (1 mM PMSF and DTT, $10 \mathrm{mg}$ / $\mathrm{ml}$ aprotinin, leupeptin and pepstatin). The cell suspension was homogenized with a Dounce homogenizer (KONTES, USA) until more than $50 \%$ of the cells were positive for trypan blue uptake, the homogenates were centrifuged at $600 \times g$ for $5 \mathrm{~min}$ and the supernatants were subjected to further centrifugation at $10000 \times$ $g$ for $10 \mathrm{~min}$. The supernatants were then subjected to further ultracentrifugation at $100000 \times \mathrm{g}$ for $45 \mathrm{~min}$. The resulting supernatants represent the cytosolic fractions.

Mouse liver mitochondrial isolation Briefly, mouse liver was suspended in $10 \mathrm{ml}$ of MIB buffer (250 mM sucrose, $2 \mathrm{mM}$ HEPES pH7.4, 0.1 mM EDTA and 0.1\% BSA) and dissociated using a $15-\mathrm{ml}$ Dounce homogenizer with a tight-fitting Teflon pestle. Mitochondria were isolated by multiple steps of centrifugation in a Sorvall centrifuge with a swinging bucket rotor. The cellular lysates were centrifuged at $600 \times g$ for $10 \mathrm{~min}$ and the supernatants were centrifuged at $10000 \times g$ for $15 \mathrm{~min}$. The mitochondrial pellets were resuspended in $15 \mathrm{ml}$ of fresh MIB, centrifuged at 1 $500 \times g$ for $5 \mathrm{~min}$ and the supernatant centrifuged at $10000 \times g$ for $10 \mathrm{~min}$. The last two steps were repeated twice. The final pellets were resuspended in $400 \mu$ of ice-cold MIB.

\section{Diagonal gels}

SCR preparation HMP (Keilin-Hartree Heart Muscle Preparation) was prepared according to the published method of Keilin and Hartree [47]. SCR was purified from HMP according to the published method [48]. Protein concentration was determined by the Bradford method.

Isolation of SCR by SDS-PAGE Diagonal gel was prepared according to the published method [39]. A volume of $50 \mu \mathrm{g}$ of SCR proteins was resolved by $12 \%$ SDS-PAGE (first dimension). After migration, the lane containing the protein was excised and soaked in $40 \% \mathrm{EtOH}$ and $10 \%$ acetic acid for $10 \mathrm{~min}$, in $30 \% \mathrm{EtOH}$ for 10 min and then in ultra pure water for $2 \times 10 \mathrm{~min}$. The lane was then air dried (15-30 min), soaked in buffer A (50 mM Tris- $\mathrm{HCl}, 150$ $\mathrm{mM} \mathrm{NaCl}, 10 \mathrm{mM}$ DTT) with or without $50 \mu \mathrm{g}$ of active recombinant casp. 3 and incubated overnight at $37^{\circ} \mathrm{C}$. The lane was washed in water to remove the excess protease and then incubated at $95{ }^{\circ} \mathrm{C}$ for $10 \mathrm{~min}$ in loading buffer $(50 \mathrm{mM}$ Tris $\mathrm{pH} 6.8,2 \% \mathrm{SDS}, 0.1 \%$ bromophenol blue, $10 \%$ glycerol, $2.5 \% \beta$-mercaptoethanol). After cooling, the lane was loaded on a second acrylamide gel $(15 \%)$ and resolved by SDS-PAGE. After migration, the gel was stained using GelCode Blue Stain Reagent (Pierce). Cleaved proteins, which are located under the diagonal, were excised from the gels and identified by MALDI-TOF-MS (AXIMA-CFR TM plus, Japan).

Assay for ROS generation and electron transport chain (ETC) enzyme activities in isolated mitochondria

ROS generation Mitochondrial ROS generation was detected using luminol plus horseradish peroxidase-derived chemiluminescence with the BPCL Ultra-weak luminescence analyzer at $37^{\circ} \mathrm{C}$. The reaction mixtures contained $500 \mu \mathrm{M}$ luminol, 2.5 units of horseradish peroxidase, $50 \mathrm{mM}$ Na-phosphate buffer $\mathrm{pH}$ 7.4, $4 \mathrm{mg} / \mathrm{ml} \mathrm{c-dHMP}$ and different concentrations of active recombinant casp. 3 in a total volume of $1 \mathrm{ml}$. The luminol plus horseradish peroxidase-derived chemiluminescence was initiated by adding $300 \mu \mathrm{M}$ NADH as substrate. The integrity of the signal peak reflects the formation of ROS. The correlation between recombinant casp. 3 concentration and ROS formations was plotted.

ETC enzyme activity (complex II-III and IV) All the assays were made at $30{ }^{\circ} \mathrm{C}$ in $50 \mathrm{mM}$ phosphate buffer (pH 7.4) using mitochondria purified on sucrose gradients. Complex II-III activity was measured by the rate of cyto.c reduction at $550 \mathrm{~nm}$ using succinate $(160 \mu \mathrm{M})$ as the electron donor. The specificity of the reaction was tested by the antimycin-A $(20 \mu \mathrm{M})$ sensitivity of the reaction. Complex IV activity was measured by the rate of cyto.c oxidation at $550 \mathrm{~nm}$ using 5-15 $\mu \mathrm{g}$ proteins and reduced cyto.c at a final concentration of $30 \mu \mathrm{M}$. We used an extinction coefficient of $19 \mathrm{M}^{-1} \cdot \mathrm{cm}^{-1}$. The specificity of the reaction was tested by the $\mathrm{KCN}$ (4 mM) sensitivity to the reaction.

\section{Determination of intracellular ROS production}

ROS production was detected using $\mathrm{CM}-\mathrm{H}_{2}$ DCFDA, an uncharged, cell-permeable fluorescent probe. When being incubated with the cells, this dye can readily diffuse into cells and is hydrolyzed by intracellular esterases to yield $\mathrm{H}_{2} \mathrm{DCF}$, which is trapped within the cells. Then, it is oxidized from the nonfluorescent form to a highly fluorescent compound by the hydrogen peroxide or other low molecular weight peroxides produced in the cells. Thus, the fluorescence intensity is proportional to the amount of peroxide produced by the cells. Exponentially growing cells $\left(1 \times 10^{5}\right.$ cells $/ \mathrm{ml}$ ) were labeled with $\mathrm{CM}-\mathrm{H}_{2}$ DCFDA (final concentration $2 \mu \mathrm{M}$ ) for $1 \mathrm{~h}$ before treatment and washed with PBS. The green fluorescence intensity in the cells was examined by FACS (Becton Dickinson, USA) with excitation at $488 \mathrm{~nm}$. Propidium iodide (PI; final concentration $10 \mathrm{ng} / \mathrm{ml}$ ) was added $1 \mathrm{~min}$ before flow cytometry. To validate the data, $0.1 \mathrm{mM} \mathrm{H}_{2} \mathrm{O}_{2}$ was added $1 \mathrm{~h}$ before the staining (not shown). The data were analyzed with the Cell Quest software (BD) for the cell population from which apoptotic cells were gated out against PI positively.

\section{Measurement of mitochondrial membrane potential}

This assay was performed as described previously [28]. Briefly, cells were collected after treated with STS. $\operatorname{DiOC}_{6}(3)$ (final concentration $10 \mathrm{nM})$ was added to $0.2 \mathrm{ml}$ cell suspension $\left(1 \times 10^{5}\right.$ cells/ $\mathrm{ml})$ in PBS (pH 7.2) and incubated at $37{ }^{\circ} \mathrm{C}$ for $5 \mathrm{~min}$. PI $(5 \mu \mathrm{lof}$ $500 \mathrm{mg} / \mathrm{ml}$ stock) was added $30 \mathrm{~s}$ before analysis. The data were validated by addition of $10 \mu \mathrm{M}$ carbonyl cyanide m-chlorophenylhydrazone (CCCP) after 5 min of DiOC6(3) loading.

\section{Oxidative phosphorylation assay}

Polarographic measurement of oxidative phosphorylation was performed as previously described [49]. Briefly, mitochondrial oxygen uptake was followed with a Clark type electrode (Yellow Springs Instruments, YSI) connected to a chart recorder via YSI Oxygen monitor. The electrode chamber was loaded with $500 \mu 1$ incubation medium (100 mM KCl, $50 \mathrm{mM}$ MOPS, $1 \mathrm{mM}$ EGTA, $5 \mathrm{mM}$ potassium phosphate, $1 \mathrm{mg} / \mathrm{ml}$ defatted BSA) and equilibrated at $30{ }^{\circ} \mathrm{C}$ before the oxygen monitor was set to $100 \%$ oxygen 
saturation. The following components were then added sequentially: mitochondria (maximum $100 \mu \mathrm{g}$ ); 25-50 nM ADP, allowing the mitochondria to consume endogenous substrates; either $10 \mathrm{mM}$ L-malate or $20 \mathrm{mM}$ succinate, providing a defined electron donor specific for complex I or II, respectively; 25-100 nM ADP to determine state 3 and state 4 respiration rates.

\section{Analysis of protein expression}

Briefly, cells were washed and lysed in buffer containing $150 \mathrm{M}$ $\mathrm{NaCl}, 25 \mathrm{mM}$ HEPES pH 7.4, 1\% CHAPS, 0.25\% sodium deoxycholate, $1 \mathrm{mM}$ EGTA, $1 \mathrm{mM}$ DTT, $50 \mu \mathrm{g} / \mathrm{ml}$ trypsin inhibitor, $1 \mathrm{mM}$ PMSF and $10 \mu \mathrm{g} / \mathrm{ml}$ aprotinin, leupeptin and pepstatin. Proteins from total cell lysates were resolved on $12 \%-15 \%$ SDS-PAGE and transferred to a nitrocellulose membrane. The membranes were blocked with $5 \%$ nonfat dry milk and $0.1 \%$ Tween 20 for $2 \mathrm{~h}$ at room temperature and incubated with the indicated antibodies at $4{ }^{\circ} \mathrm{C}$ overnight. Immune complexes were detected with HRPconjugated secondary antibody and visualized by ECL (Pierce).

\section{Flow cytometric assay for Annexin V positivity}

Apoptosis was measured using the Annexin V detection kit according to the manufacturer's instruction. Flow cytometric analysis was performed to monitor the green fluorescence of FITCconjugated Annexin V and the red fluorescence of DNA-bound PI.

\section{Cleavage of cyto.c1 protein}

Caspase in vitro cleavage experiments were performed as described previously with modification $[42,43]$. In brief, $20-50 \mu \mathrm{g}$ of cell lysate or isolated mitochondria lysate were incubated with $0-200 \mathrm{ng}$ of recombinant casp. 3 at $37^{\circ} \mathrm{C}$ for $2 \mathrm{~h}$ in $50 \mu \mathrm{l}$ of reaction buffer (100 mM Hepes pH 7.5, 20\% glycerol, $0.5 \mathrm{mM}$ EDTA, $10 \mathrm{mM}$ DTT). The reactions were stopped and resolved by $15 \%$ SDS-PAGE and western blotted with cyto.c1 antibody [40, 41].

\section{Cell fractionation and in vitro cell-free assay for caspase- induced cyto.c release}

Isolated mitochondria $(20-50 \mu \mathrm{g})$ were incubated in a total volume of $50 \mu \mathrm{l}$ buffer ( $250 \mathrm{mM}$ sucrose, $2 \mathrm{mM}$ HEPES, $0.5 \mathrm{mM}$ $\mathrm{KH}_{2} \mathrm{PO}_{4}$ and $4.2 \mathrm{mM}$ potassium succinate, $\left.\mathrm{pH} 7.4\right)$ in the presence or absence of recombinant casp. 3 for $2 \mathrm{~h}$ at $25^{\circ} \mathrm{C}$, followed by centrifugal separation of mitochondria $\left(12000 \times \mathrm{g}, 10 \mathrm{~min}\right.$ at $\left.4{ }^{\circ} \mathrm{C}\right)$. Aliquots of the supernatant $(20 \mu \mathrm{l})$ were subjected to western blotting. Cyto.c was detected by anti-cyto.c mAb. Equal protein loading was confirmed by immunodetection of cyto.c oxidase subunit IV (COX-IV) or VADC.

\section{siRNA design and transfection}

The pSilencertt ${ }^{\mathrm{TM}}$ 4.1-CMV neo vector were inserted by a DNA fragment encoding a 19-mer hairpin sequence specific to the cyto. c1 mRNA target, a loop sequence separating the two complementary domains and a dinucleotide overhang that can hybridize with the RNA target.

\#1: sense: $5^{\prime}$-gatccgctgttcgactatttcccattcaagaga tgggaaatagtcgaacagctta-3'

Anti-sense: c1-rnai1-r: 5'-agcttaagctgttcgactatttcccatctcttgaa tgggaaatagtcgaacagcg-3'

\#2: sense: $5^{\prime}$-gatccgatgttgatgatgatggct ttcaagaga agccatcatcatcaacatctta-3'

Anti-sense: $5^{\prime}$-agcttaagatgttgatgatgatgget tctcttgaa agccatcatcat- caacatcg-3'

\#3: sense: $5^{\prime}$-gatcc tgaagatggggagatgttc ttcaagaga gaacatctccccatcttcatta-3'

Anti-sense: $5^{\prime}$-agcttaatgaagatggggagatgttc tctcttgaa gaacatctccccatcttcag-3'

The $5^{\prime}$ ends of the two oligonucleotides form the $B a m \mathrm{H} 1$ and Hind III restriction site overhangs that facilitate efficient directional cloning into pSilencer 4.1-CMV neo.

HeLa cells in six-well plates were transfected with phosphate calcium method. Cells were collected and lysed with NP40 buffer for immunoblotting with anti-cyto.c1 antibody. Cells were cultured in the G418-containing medium $(200-500 \mu \mathrm{g} / \mathrm{ml})$ to enrich the cells that were successfully transfected. The population for an expected phenotype and/or the expression of the target gene was analyzed.

\section{Construction of Smac signaling peptide-tagged casp. 3}

$\mathrm{Smac}$ is a protein localized in the intermembrane space of mitochondrial. The N-terminal 55 residues serve as the mitochondria targeting sequence $[32,33]$. The DNA sequence of Smac signaling peptide targeting mitochondrial intermembrane space were fused to the N-terminal of casp.3 by PCR and cloned into pCDNA4TO/ myc.his B vector by Kpn I and Xho I.

\section{Acknowledgments}

This work was supported by grants from the National Basic Research Program (973 program project, No 2009CB521800 and 2011CB910900), the National Natural Science Foundation of China (No 30910103910) to Q Chen and 973 program project (2010CB912200) to Y Zhu. We thank Prof Richard Flavell (Yale School of Medicine, USA) for his kind supply of caspase 3-deficient MEF cells; Prof Douglas Green (St. Jude Children's Research Hospital, USA) for providing the cyto.c-GFP constructs; Prof Xiaodong Wang (University of Texas Southwestern Medical center, USA), Dr Aimin Zhou (Cleveland State University, USA), Dr Wei-Xing Zong (Stony Brook University, USA), Dr Honggang Wang (H. Lee Moffitt Cancer Center, USA) and Prof Jun Zhou (Nankai University, China) for their critical reading of the manuscript and valuable comments.

\section{References}

1 Boveris A, Chance B. The mitochondrial generation of hydrogen peroxide. General properties and effect of hyperbaric oxygen. Biochem J 1973; 134:707-716.

2 Mitchell P. Protonmotive redox mechanism of the cytochrome b-c1 complex in the respiratory chain: protonmotive ubiquinone cycle. FEBS Lett 1975; 56:1-6.

3 Brand MD, Murphy MP. Control of electron flux through the respiratory chain in mitochondria and cells. Biol Rev Camb Philos Soc 1987; 62:141-193.

4 Xia T, Jiang C, Li L, Wu C, Chen Q, Liu SS. A study on permeability transition pore opening and cytochrome c release from mitochondria, induced by caspase-3 in vitro. FEBS Lett 2002; 510:62-66.

5 Crofts AR. The cytochrome bc1 complex: function in the context of structure. Annu Rev Physiol 2004; 66:689-733.

6 Zhao Y, Wang ZB, Xu JX. Effect of cytochrome c on the gen- 
eration and elimination of $\mathrm{O} 2 *$ - and $\mathrm{H} 2 \mathrm{O} 2$ in mitochondria. $J$ Biol Chem 2003; 278:2356-2360.

7 Zhang L, Yu L, Yu CA. Generation of superoxide anion by succinate-cytochrome c reductase from bovine heart mitochondria. J Biol Chem 1998; 273:33972-33976.

8 Cape JL, Bowman MK, Kramer DM. A semiquinone intermediate generated at the Qo site of the cytochrome bc1 complex: importance for the Q-cycle and superoxide production. Proc Natl Acad Sci USA 2007; 104:7887-7892.

9 Wallace DC. A mitochondrial paradigm of metabolic and degenerative diseases, aging, and cancer: a dawn for evolutionary medicine. Annu Rev Genet 2005; 39:359-407.

10 Wallace DC. Mitochondrial diseases in man and mouse. Science 1999; 283:1482-1488.

11 Jin S, DiPaola RS, Mathew R, White E. Metabolic catastrophe as a means to cancer cell death. J Cell Sci 2007; 120:379383.

12 Yang J, Liu X, Bhalla K, et al. Prevention of apoptosis by Bcl-2: release of cytochrome $\mathrm{c}$ from mitochondria blocked. Science 1997; 275:1129-1132.

13 Green DR, Reed JC. Mitochondria and apoptosis. Science 1998; 281:1309-1312.

14 Shi Y. Apoptosome: the cellular engine for the activation of caspase-9. Structure 2002; 10:285-288.

15 Riedl SJ, Salvesen GS. The apoptosome: signalling platform of cell death. Nat Rev Mol Cell Biol 2007; 8:405-413.

16 Riedl SJ, Li W, Chao Y, Schwarzenbacher R, Shi Y. Structure of the apoptotic protease-activating factor 1 bound to ADP. Nature 2005; 434:926-933.

17 Bao Q, Riedl SJ, Shi Y. Structure of Apaf-1 in the auto-inhibited form: a critical role for ADP. Cell Cycle 2005; 4:10011003.

18 Marzo I, Susin SA, Petit PX, et al. Caspases disrupt mitochondrial membrane barrier function. FEBS Lett 1998; 427:198-202.

19 Goldstein JC, Waterhouse NJ, Juin P, Evan GI, Green DR. The coordinate release of cytochrome c during apoptosis is rapid, complete and kinetically invariant. Nat Cell Biol 2000; 2:156-162.

20 Cepero E, King AM, Coffey LM, Perez RG, Boise LH. Caspase-9 and effector caspases have sequential and distinct effects on mitochondria. Oncogene 2005; 24:6354-6366.

21 Bossy-Wetzel E, Green DR. Caspases induce cytochrome c release from mitochondria by activating cytosolic factors. $J$ Biol Chem 1999; 274:17484-17490.

22 Annis MG, Soucie EL, Dlugosz PJ, et al. Bax forms multispanning monomers that oligomerize to permeabilize membranes during apoptosis. EMBO J 2005; 24:2096-2103.

23 Ott M, Gogvadze V, Orrenius S, Zhivotovsky B. Mitochondria, oxidative stress and cell death. Apoptosis 2007; 12:913922.

24 Orrenius S, Gogvadze V, Zhivotovsky B. Mitochondrial oxidative stress: implications for cell death. Annu Rev Pharmacol Toxicol 2007; 47:143-183.

25 Chandra D, Tang DG. Mitochondrially localized active caspase- 9 and caspase- 3 result mostly from translocation from the cytosol and partly from caspase-mediated activation in the organelle. Lack of evidence for Apaf-1-mediated procaspase-9 activation in the mitochondria. J Biol Chem 2003; 278:17408-
17420.

26 Ricci JE, Waterhouse N, Green DR. Mitochondrial functions during cell death, a complex (I-V) dilemma. Cell Death Differ 2003; 10:488-492.

27 Ricci JE, Gottlieb RA, Green DR. Caspase-mediated loss of mitochondrial function and generation of reactive oxygen species during apoptosis. J Cell Biol 2003; 160:65-75.

28 Chen Q, Gong B, Almasan A. Distinct stages of cytochrome c release from mitochondria: evidence for a feedback amplification loop linking caspase activation to mitochondrial dysfunction in genotoxic stress induced apoptosis. Cell Death Differ 2000; 7:227-233.

29 Chen Q, Chai YC, Mazumder S, et al. The late increase in intracellular free radical oxygen species during apoptosis is associated with cytochrome c release, caspase activation, and mitochondrial dysfunction. Cell Death Differ 2003; 10:323334.

30 Sun F, Huo X, Zhai Y, et al. Crystal structure of mitochondrial respiratory membrane protein complex II. Cell 2005; 121:1043-1057.

31 Poot M, Zhang YZ, Kramer JA, et al. Analysis of mitochondrial morphology and function with novel fixable fluorescent stains. J Histochem Cytochem 1996; 44:1363-1372.

32 Du C, Fang M, Li Y, Li L, Wang X. Smac, a mitochondrial protein that promotes cytochrome c-dependent caspase activation by eliminating IAP inhibition. Cell 2000; 102:33-42.

33 Chai J, Du C, Wu JW, Kyin S, Wang X, Shi Y. Structural and biochemical basis of apoptotic activation by Smac/DIABLO. Nature 2000; 406:855-862.

34 Xia D, Yu CA, Kim H, et al. Crystal structure of the cytochrome bc1 complex from bovine heart mitochondria. Science 1997; 277:60-66.

35 Pletjushkina OY, Lyamzaev KG, Popova EN, et al. Effect of oxidative stress on dynamics of mitochondrial reticulum. Biochim Biophys Acta 2006; 1757:518-524.

36 Kagan VE, Tyurin VA, Jiang J, et al. Cytochrome c acts as a cardiolipin oxygenase required for release of proapoptotic factors. Nat Chem Biol 2005; 1:223-232.

37 Suto D, Sato K, Ohba Y, Yoshimura T, Fujii J. Suppression of the pro-apoptotic function of cytochrome $\mathrm{c}$ by singlet oxygen via a haem redox state-independent mechanism. Biochem $J$ 2005; 392:399-406.

38 Abdullaev Z, Bodrova ME, Chernyak BV, et al. A cytochrome c mutant with high electron transfer and antioxidant activities but devoid of apoptogenic effect. Biochem J 2002; 362:749754.

39 Jiang S, Cai J, Wallace DC, Jones DP. Cytochrome c-mediated apoptosis in cells lacking mitochondrial DNA. Signaling pathway involving release and caspase 3 activation is conserved. J Biol Chem 1999; 274:29905-29911.

40 Marchetti P, Susin SA, Decaudin D, et al. Apoptosis-associated derangement of mitochondrial function in cells lacking mitochondrial DNA. Cancer Res 1996; 56:2033-2038.

41 Ricci JE, Munoz-Pinedo C, Fitzgerald P, et al. Disruption of mitochondrial function during apoptosis is mediated by caspase cleavage of the p75 subunit of complex I of the electron transport chain. Cell 2004; 117:773-786.

42 Weng C, Li Y, Xu D, Shi Y, Tang H. Specific cleavage of Mcl1 by caspase-3 in tumor necrosis factor-related apoptosis-in- 
ducing ligand (TRAIL)-induced apoptosis in Jurkat leukemia T cells. J Biol Chem 2005; 280:10491-10500.

43 Cheng EH, Kirsch DG, Clem RJ, et al. Conversion of Bcl2 to a Bax-like death effector by caspases. Science 1997; 278:1966-1968.

44 Mancuso M, Filosto M, Stevens JC, et al. Mitochondrial myopathy and complex III deficiency in a patient with a new stopcodon mutation (G339X) in the cytochrome b gene. J Neurol Sci 2003; 209:61-63.

45 Borisov VB. Defects in mitochondrial respiratory complexes III and IV, and human pathologies. Mol Aspects Med 2002; 23:385-412.
46 Acin-Perez R, Bayona-Bafaluy MP, Fernandez-Silva P, et al. Respiratory complex III is required to maintain complex I in mammalian mitochondria. Mol Cell 2004; 13:805-815.

47 Keilin D, Hartree EF. Activity of the cytochrome system in heart muscle preparations. Biochem J 1947; 41:500-502.

48 Yu CA, Yu L, King TE. Soluble cytochrome b-c1 complex and the reconstitution of succinate-cytochrome c reductase. $J$ Biol Chem 1974; 249:4905-4910.

49 Poe M, Gutfreund H, Estabrook RW. Kinetic studies of temperature changes and oxygen uptake in a differential calorimeter: the heat of oxidation of NADH and succinate. Arch Biochem Biophys 1967; 122:204-211.

(Supplementary information is linked to the online version of the paper on the Cell Research website.) 